\title{
The effect of induced social interaction on positive and negative affect
}

\author{
CURTIS W. MCINTYRE, DAVID WATSON, and LEE ANNA CLARK \\ Southern Methodist University, Dallas, Texas \\ and \\ STEPHEN A. CROSS \\ University of Texas at Dallas, Dallas, Texas
}

\begin{abstract}
The effects of two induced social interactions (Getting Acquainted and Lunching Together) on positive affect (PA) and negative affect (NA) were investigated with the use of the 20 -item positive and negative affect schedule (PANAS). In two experiments, undergraduate subjects completed two cycles of 9 PANASs. Each cycle contained three sets of three PANASs, which were completed during successive class periods: One set each was completed during the class period preceding, including, and following the social interaction. Within each set of three PANASs, one was completed at the beginning of a 2-h class, a second was completed $1 \mathrm{~h}$ into the class (before the social interaction), and a third was completed at the end of class (after the social interaction). One of the two 9-assessment cycles involved getting acquainted with a previously unknown classmate for $20 \mathrm{~min}$; the other involved having lunch with this classmate 2 weeks later. The resulting PA and NA scores were compared separately for each social interaction by using within-subjects analyses of variance and subsequent multiple-comparison tests. These analyses revealed that PA increased significantly after social interaction and returned to normal by the beginning of the next class period and that NA was not changed by social interaction. The implications of these results are discussed.
\end{abstract}

Extensive recent research indicates that two broad factors, positive affect and negative affect, represent the major dimensions of mood (Mayer \& Gaschke, 1988; Tellegen, 1985; Watson, 1988b; Watson \& Tellegen, 1985). Positive affect (PA) reflects a person's level of pleasurable engagement with the environment. High PA is a state of enthusiasm, energy, mental alertness, and determination, whereas low PA is best characterized by feelings of lassitude and lethargy. In contrast, negative affect (NA) reflects a person's general level of subjective distress. High NA is an aversive mood state that includes feelings of anger, guilt, fear, tension, sadness, scorn, and disgust, whereas low NA is best characterized by calmness and serenity.

PA and NA are highly distinctive mood dimensions that are largely independent of one another. Their distinctive characteristics have emerged gradually from studies showing that they are related to fundamentally different classes of variables. For example, variations in NA - but not PAare broadly associated with somatic complaints, psychopathology, and self-reported stress. In contrast, PA-but not NA -is related to diverse indicators of social activity and interpersonal satisfaction. Moreover, these patterns have emerged consistently from both between-subjects (Beiser, 1974; Bradburn, 1969; Phillips, 1967; Watson,

Correspondence should be addressed to Curtis W. McIntyre, Department of Psychology, Southern Methodist University, Dallas, TX 75275. 1988a; Watson \& Pennebaker, 1989) and within-subjects (Clark \& Watson, 1988; McIntyre, Watson, \& Cunningham, 1990; Watson, 1988a) analyses.

The current study further examines the relationship between mood and social activity. As noted previously, existing research has linked social interaction strongly with both intra- and interindividual differences in PA. However, the meaning of this general relationship between PA and social activity is not clear. In previous studies, PA generally has been examined in relation to naturally occurring social interactions. Thus, it is not clear which variable affects the other; that is, does high PA lead to increased social activity, or does social activity result in higher PA?

Existing research indicates that both of these explanations may be true. For example, high levels of PA (either naturally occurring or experimentally induced) are associated with enhanced affiliative feelings and an increased preference for social and prosocial activities (Cunningham, 1988b; Rossi \& Rossi, 1977; Strickland, Hale, \& Anderson, 1975). Moreover, elevated PA is related to increases in the frequency of social and prosocial behavior (Cunningham, 1988a; Cunningham, Steinberg, \& Grev, 1980; Shaffer \& Smith, 1985).

Conversely, Clark and Watson (1988) found that some types of social events were much more pleasurable than others. More specifically, epicurean, informal, and physical social activities (i.e., eating and drinking with others, parties, skiing, etc.) were associated strongly with in- 
creased PA, whereas formal and/or sedentary social events (club meetings, lessons, conversations with others, etc.) were unrelated to mood. Given these results, it is plausible that some types of social activities raise PA, at least temporarily.

To date, however, no study has examined the effects of experimentally induced social interaction on PA and NA. The present study extends previous research by examining the immediate, short-term effects of two types of experimentally induced social activity-getting acquainted with a stranger and lunching together with a casual acquaintance-on measures of these mood dimensions. The PA and NA scales from the positive and negative affect schedule (PANAS; Watson, Clark, \& Tellegen, 1988) were used: first, to establish baseline mood levels; second, to assess changes from these baseline levels immediately after the social interactions; and third, to examine when mood returned to its baseline level. On the basis of previous research (e.g., Clark \& Watson, 1988; McIntyre, Watson, \& Cunningham, 1990), we hypothesized that both types of social activity would increase PA but would not impact NA. More specifically, we predicted that PA would reach its zenith immediately after the social interaction and that the increase in PA would not be longlasting, so that PA would return by the following day to its premanipulation, baseline level. In contrast, we expected that NA would not vary as a function of social activity.

\section{EXPERIMENT 1: GETTING ACQUAINTED}

The social interaction used in Experiment 1 required subjects to get acquainted with a classmate they had not previously met. The subjects were given the last $20 \mathrm{~min}$ of a regular class period to get acquainted with one another. Measures of PA and NA also were obtained at these same times during the class period in which the subjects got acquainted. This allowed the measurement of PA and NA immediately before and after social interaction. In addition, to provide complete baseline mood measures, PA and NA were assessed at these same times during class on the days before and after the subjects engaged in this activity.

\section{Method}

Subjects. Sixty undergraduates ( 30 male and 30 female) served as subjects. Their mean age was 20.23 years.

Measures. The PANAS (Watson et al., 1988) contains 10-item PA and NA scales. The 20 mood descriptors were selected on the basis of several factor analyses and are good factor markers of either high PA or high NA. Watson et al. (1988) report extensive data demonstrating the reliability and validity of these scales.

The PANAS PA scale consists of active, alert, attentive, determined, enthusiastic, excited, inspired, interested, proud, and strong. The PANAS NA scale consists of afraid, ashamed, distressed, guilty, hostile, irritable, jittery, nervous, scared, and upset. Subjects rated the extent to which they were experiencing each mood descriptor at that moment. These ratings were made using a 5-point scale: $1=$ very slightly or not at all, $2=$ a little, $3=$ moderately, $4=$ quite $a$ bit, and $5=$ very much. Total NA and PA scores were obtained by summing the ratings made for the 10 relevant mood descriptors. Thus, the possible range of scores for each affect was 10 to 50 .

Design and Procedure. Each subject completed the PANAS on 9 separate occasions, divided into three sets of three. The first set of three
Table 1

Getting Acquainted: Mean Positive and Negative Affect Scores

\begin{tabular}{lcccc}
\hline & \multicolumn{4}{c}{ Class Time } \\
\cline { 2 - 5 } \multicolumn{1}{c}{ Condition } & Begin & Hour Into & End & Mean \\
\hline & \multicolumn{1}{c}{ Positive } & Affect & & \\
Presocial interaction & 24.18 & 23.23 & 25.87 & 24.43 \\
Social interaction & 21.83 & 22.00 & 27.85 & 23.89 \\
Postsocial interaction & 22.48 & 22.17 & 23.08 & 22.58 \\
Mean & 22.83 & 22.47 & 25.60 & 23.63 \\
& Negative & Affect & & \\
Presocial interaction & 12.72 & 11.78 & 12.13 & 12.21 \\
Social interaction & 12.37 & 12.10 & 11.68 & 12.05 \\
Postsocial interaction & 12.45 & 12.03 & 12.15 & 12.21 \\
Mean & 12.51 & 11.94 & 11.99 & 12.16 \\
\hline
\end{tabular}

PANASs was completed at the beginning, $1 \mathrm{~h}$ into, and at the end of the 2-h class preceding the social interaction. This set provided presocial interaction baseline scores for PA and NA. The second set of three PANASs was completed at the beginning of class, $1 \mathrm{~h}$ into the class (about $30 \mathrm{~min}$ before the social interaction), and at the end of class (immediately after the social interaction). This set provided the pre- and postsocial interaction scores for PA and NA. The third set of three PANASs was completed at the beginning, $1 \mathrm{~h}$ into, and at the end of the 2-h class following the social interaction. This set provided postsocial interaction baseline scores for PA and NA.

\section{Results}

The basic data were the PA and NA scores obtained for each subject at each of the nine assessments. The mean PA and NA scores are presented in Table 1. Application of separate 3 conditions (presocial interaction, social interaction, and postsocial interaction) $\times 3$ times (at the beginning, $1 \mathrm{~h}$ into, and at the end of the 2-h class) withinsubjects analyses of variance to the PA and NA data revealed the following results.

Positive affect. For PA, we found a significant main effect for conditions $[F(2,118)=5.37, p<.01]$, a significant main effect for times $[F(2,118)=14.16, p<$ $.03]$, and a significant conditions $\times$ times interaction $[F(4,236)=7.40, p<.01]$. Subsequent Newman-Keuls multiple-comparison tests $(p<.05)$ contrasting the condition means revealed that the presocial interaction mean (24.43) was significantly greater than the social interaction mean (23.89), which, in turn, was significantly greater than the postsocial interaction mean (22.58). Contrasting the times means, the mean obtained at the end of class (25.60) was significantly greater than the means obtained at the beginning of class (22.83) and $1 \mathrm{~h}$ into class (22.47). Subsequent Cicchetti (1972) multiple-comparison tests $(p<.05)$ contrasting the conditions $\times$ times interaction means indicated that the mean (27.85) obtained immediately after the social interaction (Getting Acquainted) was significantly greater than the other means, which did not differ significantly from each other.

Negative affect. For NA, only a significant main effect for times was found $[F(2,118)=5.00, p<.01]$. Subsequent Newman-Keuls multiple-comparison tests $(p<$ .05 ) revealed that the mean obtained at the beginning of class (12.51) was significantly greater than the means obtained $1 \mathrm{~h}$ into (11.97) and at the end of class (11.99). 


\section{Discussion}

As expected, the social interaction of getting acquainted with a previously unknown classmate significantly increased PA immediately after the interaction, as can be seen by comparing the mean obtained after social interaction with all the other means. Although this increase in PA is both substantial (5.85) and robust, it is short-lived. Clearly, it has dissipated by the beginning of the next class meeting (within $24 \mathrm{~h}$ ). Also as expected, the social interaction of getting acquainted with a previously unknown classmate had no effect on NA.

\section{EXPERIMENT 2: LUNCHING TOGETHER}

The social interaction used in Experiment 2 required the subjects to have lunch with the same classmate with whom they had gotten acquainted earlier in the semester. The subjects were given the last $50 \mathrm{~min}$ of a regular class to have lunch with this classmate. As before, measures of PA and NA were obtained three times on the day the subjects had lunch together. This allowed the measurements of PA and NA before and immediately after the social interaction. Also, as before, PA and NA were assessed at those same times during class on the days before and after the subjects had lunch together.

\section{Method \\ Subjects. Fifty-four of the 60 undergraduates used in Experiment 1 (24 male and 30 female) served as subjects in Experiment 2. \\ Measures, Design, and Procedure. The measures, design, and proce- dure were the same as in Experiment 1, except that the social interaction in Experiment 2 involved having lunch with the same classmate with whom they had gotten acquainted earlier in the semester. As before. each subject completed the PANAS on 9 separate occasions, divided into three sets of three assessments.}

\section{Results}

The basic data were the PA and NA scores obtained for each subject at each of the nine assessments. The mean PA and NA scores are presented in Table 2. Application of separate 3 conditions (presocial interaction, social interaction, and postsocial interaction) $\times 3$ times (at the beginning, $1 \mathrm{~h}$ into, and at the end of the 2 -h class) within-

Table 2

Lunching Together: Mean Positive and Negative Affect Scores

\begin{tabular}{lcccc}
\hline \multirow{2}{*}{\multicolumn{1}{c}{ Condition }} & \multicolumn{4}{c}{ Class Time } \\
\cline { 2 - 5 } & Begin & Hour Into & End & Mean \\
\hline & Positive & Affect & & \\
Presocial interaction & 21.93 & 23.04 & 23.83 & 22.93 \\
Social interaction & 23.02 & 22.54 & 26.80 & 24.12 \\
Postsocial interaction & 22.17 & 21.71 & 23.96 & 22.62 \\
Mean & 22.37 & 22.43 & 24.86 & 23.22 \\
& Negative & Affect & & \\
Presocial interaction & 12.83 & 12.19 & 12.19 & 12.40 \\
Social interaction & 12.22 & 12.19 & 11.37 & 11.93 \\
Postsocial interaction & 11.96 & 12.11 & 12.41 & 12.16 \\
Mean & 12.34 & 12.16 & 11.99 & 12.16 \\
\hline
\end{tabular}

subjects analyses of variance to the PA and NA data yielded the following results.

Positive affect. For PA, a significant main effect for times $[F(2,106)=10.35, p<.01]$ and a significant conditions $\times$ times interaction $[F(4,212)=2.85, p<.03]$ were found. Subsequent Newman-Keuls multiple-comparison tests $(p<.05)$ revealed the following: Contrasting the times means, the mean obtained at the end of class (24.86) was significantly greater than the means obtained at the beginning of class (22.37) and $1 \mathrm{~h}$ into class (22.43). Subsequent Cicchetti (1972) multiple-comparison tests $(p<$ .05 ) contrasting the condition $\times$ times interaction means indicated that the mean obtained immediately after the social interaction (26.80) was significantly greater than the other means, which did not differ significantly from each other.

Negative affect. For NA, no significant main effects or interactions were found.

\section{Discussion}

Replicating the findings of Experiment 1, the social interaction of having lunch with a casual acquaintance significantly increased PA, as indicated by the higher mean PA score obtained after this social interaction, compared with all the other means. As in Experiment 1, this increase in PA was substantial (4.26), but also short-lived, dissipating by the beginning of the next class meeting (within $24 \mathrm{~h}$ ). Also as expected, the social interaction of having lunch with a casual acquaintance had no effect on NA.

\section{CONCLUSIONS}

As discussed earlier, several studies, employing both between- and within-subjects designs, have demonstrated that PA is related consistently to diverse measures of naturally occurring social activity. Replicating and extending the results of this earlier research, the current studies show that two different types of experimentally induced social interaction (Getting Acquainted and Lunching Together) lead to transient increases in PA but not NA. Clearly, the relationship between PA and social interaction is both broad and robust.

Whereas the current data offer strong evidence that normal social activity produces transient elevations in PA, other research also indicates that high levels of PA (either naturally occurring or experimentally induced) lead to an increased preference for social activity, as well as to actual increases in the frequency of social and prosocial behavior (e.g., Cunningham, 1988a, 1988b; Shaffer \& Smith, 1985; Strickland et al., 1975). Thus, PA and interpersonal activity may mutually influence each other. That is, normal types of social interaction ordinarily lead to elevated levels of PA; conversely, elevated PA also increases the desire for-and frequency of-social behavior. In other words, increased PA is both a cause and a result of social behavior. On the basis of these and other data, Watson and Clark (in press) have argued that PA and so- 
cial interaction ultimately may reflect different components of a single, integrated system that regulates the occurrence of active, pleasure-seeking behavior (see also Tellegen, 1985). Similarly, Depue, Krauss, and Spoont (1987) have suggested that PA and interpersonal activity both reflect "a goal-directed activation system which involves, and supports, behavioral mastery of the social and work environment"' (p. 105). These models clearly go beyond the available data but offer a useful guide for future research.

In contrast, NA levels are not affected by the occurrence or nonoccurrence of active, pleasurable social experiences-at least not by the experimentally induced social activities used in the two current experiments. Instead, NA is related to major crises and stressful eventsfor example, health problems, evaluation situations, embarrassments, and failures (see McIntyre et al., 1990; Watson \& Clark, 1984; Watson \& Pennebaker, 1989). Conversely, these crises and stressors do not affect PA significantly. Hence, one can lead an active and interesting life, and experience high levels of PA, even when confronted with considerable stresses and strains (Watson, 1988a; Watson \& Pennebaker, 1989). Clearly, these findings illustrate the distinctive qualities of PA and NA, as well as demonstrate the utility of measuring these mood dimensions separately.

\section{REFERENCES}

BEISER, M. (1974). Components and correlates of mental well-being. Journal of Health \& Social Behavior, 15, 320-327.

BRADBURN, N. M. (1969). The structure of psychological well-being. Chicago: Aldine.

CicchetTI, D. V. (1972). Extension of multiple-range tests to interaction tables in the analysis of variance: A rapid approximate solution. Psychological Bulletin, 77, 405-408.

Clark, L. A., \& Watson, D. (1988). Mood and the mundane: Relations between daily life events and self-reported mood. Journal of Personality \& Social Psychology, 54, 296-308.

Cunningham, M. R. (1988a). Does happiness mean friendliness? Induced mood and heterosexual self-disclosure. Personality \& Social Psychology Bulletin, 14, 283-297.

Cunningham, M. R. (1988b). What do you do when you're happy or blue? Mood, expectancies, and behavioral interest. Motivation \& Emotion, 12, 309-331.

Cunningham, M. R., Steinberg, J., \& Grev, R. (1980). Wanting to and having to help: Separate motivations for positive mood and guilt-induced helping. Journal of Personality \& Social Psychology, 38, 181-192.

Depue, R. A., Krauss, S. P., \& Spoont, M. R. (1987). A twodimensional threshold model of seasonal bipolar affective disorder. In D. Magnusson \& A. Ohman (Eds.), Psychopathology: An interactional perspective (pp. 95-123). Orlando, FL: Academic Press.

MAYER, J. D., \& GASChKE, Y. N. (1988). The experience and metaexperience of mood. Journal of Personality \& Social Psychology, 55, $102-111$.

McIntyre, C. W., Watson, D. \& Cunningham, A. C. (1990). The effects of social interaction, exercise, and test stress on positive and negative affect. Bulletin of the Psychonomic Society, 28, 141-143.

PHILlips, D. L. (1967). Social participation and happiness. American Journal of Sociology, 72, 479-488.

Rossi, A. S., \& Rossi, P. E. (1977). Body time and social time: Mood patterns by menstrual cycle phase and day of the week. Social Science Research, 6, 273-308.

ShAFFER, D. R., \& SMITH, J. (1985). Effects of preexisting moods on observers' reactions to helpful and nonhelpful models. Motivation \& Emotion, 9, 101-122.

Strickland, B. R., Hale, W. D., \& ANDERSON, L. K. (1975). Effect of induced mood states on activity and self-reported affect. Journal of Consulting \& Clinical Psychology, 43, 587.

Tellegen, A. (1985). Structures of mood and personality and their relevance to assessing anxiety, with an emphasis on self-report. In A. H. Tuma \& J. D. Maser (Eds.), Anxiety and the anxiety disorders (pp. 681-706). Hillsdale, NJ: Erlbaum.

WATSON, D. (1988a). Intraindividual and interindividual analyses of positive and negative affect: Their relation to health complaints, perceived stress, and daily activities. Journal of Personality \& Social Psychology, 54, 1020-1030.

WATSON, D. (1988b). The vicissitudes of mood measurement: Effects of varying descriptors, time frames, and response formats on measures of positive and negative affect. Journal of Personality \& Social Psychology, 55, 128-141.

Watson, D., \& Clark, L. A. (1984). Negative affectivity: The disposition to experience aversive emotional states. Psychological Bulletin, 96, 465-490.

Watson, D., \& Clark, L. A. (in press). Extraversion and its positive emotional core. In S. Briggs, R. Hogan, \& W. Jones (Eds.), Handbook of personality psychology. San Diego: Academic Press.

Watson, D., Clark, L. A., \& Tellegen, A. (1988). Development and validation of brief measures of positive and negative affect: The PANAS scales. Journal of Personality \& Social Psychology, 54, 1063-1070.

Watson, D., \& Pennebaker, J. W. (1989). Health complaints, stress, and distress: Exploring the central role of negative affectivity. Psychological Review, 96, 234-254.

WAtson, D., \& TEllegen, A. (1985). Toward a consensual structure of mood. Psychological Bulletin, 98, 219-235.

(Manuscript received July 26, 1990.) 